\title{
Periosteal Osteosarcoma
}

National Cancer Institute

\section{Source}

National Cancer Institute. Periosteal Osteosarcoma. NCI Thesaurus. Code C8970.

An intermediate grade malignant bone-forming mesenchymal neoplasm with

chondroblastic differentiation. It arises from the surface of the bone and affects the diaphysis or diaphyseal- metaphyseal portion of the long bones. A painless mass or swelling is the most common clinical sign. It is associated with a better prognosis than conventional osteosarcoma. 